\title{
Efficient CAI in College Foreign Language Teaching
}

\author{
Jia Suo
}

\author{
Department of Foreign Languages, North China Electric Power University, Baoding, Hebei, China
}

\begin{abstract}
CAI has revolutionized the traditional educational concept and styles and contributed to the increase of teaching efficiency and teaching quality. But excessive usage of CAI will weaken the teaching effect. Therefore we can only put it in the position of "aid", and integrate it with traditional teaching by adopting the combined form of student's autonomic learning in multimedia and network environment, teacher's guidance and face-to-face teaching in classroom.
\end{abstract}

Keywords-CAI, Teacher's guidance, Face-to-face teaching in classroom

\section{计算机如何有效地辅助大学外语教学}

\author{
索佳 \\ 华北电力大学外国语学院, 保定, 河北, 中国
}

\begin{abstract}
摘 要 计算机辅助教学给传统的教育理念和教学模式带来了巨大冲击, 提高了教学效率和教学质量。但是过度依赖多媒体、网 络会削弱教学效果。因此, 多媒体、网络技术在教学中只能处于“辅助”地位, 教师依然处于“主导”地位。学生在多媒体、网络环境下 的自主学习必须和教师的课堂面授有机结合。
\end{abstract}

关键词 计算机辅助教学, 教师主导, 课堂面授

\section{1. 引言}

信息与通讯技术的发展和进步不仅改变了人们的娱乐 和交际方式, 还为学习方式和教学模式的改革提供了有力 的技术支撑。如何充分利用现代信息技术为学习服务, 是 所有师生在网络时代所要解决的问题。计算机辅助教学应 运而生。计算机辅助教学是指利用计算机和多媒体辅助手 段, 将声音、文字和图像有机地结合起来去开展教学工作。 其教学内容不再局限于课本, 不但有功能强大的教学软件, 还有海量的网络学习资源可以使用。教学结构也发生了根 本的变化, 由原来的以教师“教”为主, 转变为以学生“学” 为主。倡导学生根据自身特点和需要, 利用多媒体、网络 环境, 主动地、有针对性地进行自主学习。

\section{2. 多媒体、网络教学的优点}

多媒体、网络技术应用于英语听说教学能为其带来诸 多优势, 具体如下:

1、多媒体技术提供了生动丰富的文字、图像、声音和 影像等多种媒体的组合, 化抽象为具体, 使教学变得生动
新颖, 从而吸引学生的注意力, 培养和激发学生的兴趣; 充分刺激学生的五官, 加强学生听觉输入的效果, 增强学 生的记忆力。教育心理学家的实验发现: 对学习者分别采 用口头传播、令其默诵学习和视听传播这三种方式, 学习 结束三小时后学习者的记忆率分别为: $60 \% 、 70 \% 、 90 \%$; 三天后的记忆保持率分别为 $15 \% 、 40 \% 、 75 \%$ [1]。这一结 果表明, 多媒体教学有利于学生对所学知识的掌握。

2、网络提供了巨大的资源库和与教学软件相关的各种 链接, 扩大了学生的语言输入。Krashen 认为只有为语言学 习者提供大量可以理解的语言输入, 其中包含略高于其现 有语言水平(即 $i+1$ ) 的语言输入, 才能对学习者的语言发 展产生积极作用[2]。学生可以利用这些资源听到不同年龄, 不同职业, 不同身份的人在不同场合的对话, 置身于丰富 的语言交际环境中, 可以直接把语篇与实际的交际过程联 系起来, 不断提高自身的英语交际能力。另外, 学生如果 遇到问题, 可以随时利用这些资源和便捷的检索功能来找 到答案或解决办法, 这激发了他们的主观能动性。

3、网络提供的小组协作机制和即时通讯工具为学生提 供了充分练习英语口语表达的环境。电子邮件、论坛、BBS、 
ICQ 等方式使学生可以与世界各地的人进行交流, 新型的 网络技术还实现了多方视频实时通话。Swain 认为成功的 二语学习者既需要大量的可理解输入, 又需要语言输出[3]。 语言输出可以增强语言的准确和流利。输出可以促进二语 习得, 也可以增强输入对二语习得的作用。

4、网络提供的分级学习模式将水平各异的学生区别对 待, 有助于调动学生的学习积极性, 有利于提高其听力技 能。传统课堂里, 老师根据大部分学生的听力水平安排教 学内容, 不得不忽视学生水平的差异性, 也无法及时纠正 学生在听力细节上出现的一些问题, 削弱了部分学生的学 习积极性。而在网络环境下, 学生可以根据教学目标和自 己的水平, 自由选择听力材料的种类、内容, 难度、速度、 数量和收听频数; 听不懂某些细节时, 可以及时查看文字 资料。Peck 发现当学生有权决定听什么和怎么听的时候, 他们的学习兴趣提高了, 对自己的学习也更加负责 [4]。

5、网络提供的监测、交流答疑板块和即时通讯工具有 助于教师加强与学生的交流, 加深对学生的了解, 对学生 进行有针对性的指导, 兼顾了个体差异, 尤其是使那些在 传统课堂中容易被忽略的基础较弱的学生得到了更多关 注, 得以增强自信心, 进而取得显著的进步。

6、网络环境下学生焦虑的减轻有助于其听说能力的提 高。Krashen 认为, 情感因素对语言学习有着重要的影响[2]。 其中, 焦虑是语言学习的障碍。学习者自尊心的强弱和对 学习过程中出现的模糊现象的宽容程度等都与焦虑程度有 关。学生在网络环境下进行听说练习时, 不用害怕因为听 不懂或说错了而在同学面前丢脸, 也不用害怕因为听不懂 而跟不上全班的进度。

\section{3. 多媒体、网络教学的局限性}

但是教师也不能忽视多媒体、网络教学的局限性。

1、网络课堂缺乏传统课堂里教师的组织、控制和管理, 导致部分学生不能有效地利用网络进行自主学习, 因为很 多学生不具备完全的自主学习能力, 他们在学习过程中容 易陷入对细节的关注而忘记了学习的主题; 容易迷失在网 络海量的资源里, 走马观花, 不能进行深入的学习; 缺乏 自制能力, 不能保质保量地完成学习任务。

2、网络课堂缺乏传统课堂里师生面对面的情感交流。 教师的讲解是一门语言艺术, 在任何时候, 都具有极其重 要的作用, 是任何教学手段所无法替代的。教师的一个微 笑、一个手势或一个简单的动作在提高教学效果中都起着 重要的作用。而这是计算机无法提供的。

3、过度使用计算机不利于抽象思维能力的培养, 因为计 算机能把抽象问题形象化。英语是一门特别需要抽象思维能 力的学科, 抽象思维能力的削弱不利于对英语的深入学习。

\section{4. 计算机辅助英语听说教学的实施方法}

因此, 教师应该将基于多媒体、网络环境的教学模式 与课堂教学模式结合起来, 将 “人机对话”与“人际对话”结 合起来, 在突出学生自主学习的同时, 更不能忽视教师在 整个教学中的主导作用, 实现不同教学模式优劣势的互补, 扬长避短, 避免单一教学模式的种种弊端, 实现高效的计 算机辅助英语听说教学。具体方法如下:

1、在网络自主学习开始前教师向学生介绍多媒体教学 环境, 包括硬件环境、教学软件及操作方法、FAQ 的重点 说明等, 讲解如何在海量的数据库里搜寻并合理利用所需 的信息。

2、O'Dell 认为学生能否有效地在自学中心进行学习取 决于教师对中心设施及教学模式的熟悉程度 [5]。所以为了 使学生能够充分利用学习资源进行自主学习, 教师应掌握 计算机和网络技术基础知识, 能够熟练运用电脑及网络工 具, 熟悉多媒体教学环境和教学内容的特殊传递方式, 熟 悉学生自主学习模式下所使用的教学软件内容, 对整个教 学资源、设备有整体的认识、规划和整合的能力。

3、多媒体、网络听说教学的顺利开展需要有一个包括 教师、教辅人员及多媒体教室技术维护人员在内的配合默 契的团队。团队成员分工合作, 对学习资料进行搜集、分 类和不断的更新; 还要定期研讨, 集思广益、交流经验, 以便更好地开展多媒体、网络听说教学。

4、充分利用网络的优势, 实行分级教学, 做到因材施 教。教师指导学生进行自我反思以确定目前的听力水平, 找出听力中的薄弱环节, 然后据此设定明确的阶段性学习 目标并制订一份切实可行的听力训练计划, 包括材料选择 和时间安排等。听力材料要难度适中, 既符合学生当前的 水平又要有一定难度。

5、教师要利用课堂面授的机会, 为学生讲解听力学习 策略, 解题思路和技巧, 并帮他们调适情感, 完成计算机 所不能完成的任务。

6、学生了解了一定的策略和技巧后, 教师制定每个阶 段的学习内容和目标, 监督和指导学生在网络课堂通过练 习去应用这些策略和技巧, 及时解答他们在学习过程中遇 到的各种问题。在此过程中教师把学生分为高、中、低三 个级别, 为其设置不同的任务, 在不同级别之内再分组, 以小组为单位共同完成任务。研究发现, 在小组讨论中, 学习者更愿意发言[6]。小组成员之间可以交流经验, 互相 帮助。这既可以使学生相互监督, 弥补了网络环境下教师 对学生监控能力下降的不足, 又可以激发其学习兴趣。

7、教师组织对学生的网络学习进行多种形式的评价, 如教师评价、学生自评或学生互评, 设计评价内容, 最终 获得不同评价人员所给出评价结果的加权值, 把它作为学 
生的平时成绩。学生成为了评价主体, 有利于提高他们学 习的积极性。网络为这种评价机制提供了平台, 使师生可 以查看学生的学习记录, 如: 学习时间, 作业完成情况, 阶段性测验成绩, 等级测试完成情况等。这些可以帮助学 生及时了解学习情况, 调整学习进度, 调整学习过程中所 采用的策略, 发现听说中存在的问题并及时纠正, 让学生 更加注重平时的听说练习, 而不是平时不练, 只要最后一 次考试通过就行了, 从而大大提高自身学习的责任感, 促 进自主能力的培养。教师也可以根据这些学习记录向学生 提出一些关于阶段性学习内容, 学习进度及学习方法的建 议。

8、由于听说课时不足, 所以教师要遵照循序渐进的原 则为学生布置课外任务。仍然要求学生以小组为单位共同 完成任务, 以小组为单位向老师汇报学习情况。

9、教师随时通过课程聊天室、网络教学平台课程讨论 区或 QQ、MSN, E-mail 来和学生交流, 帮助学生解决问 题。

\section{5. 结论}

计算机辅助教学是一门新的教育技术, 它从根本上改 变了传统的教育理念和教育方式, 有助于教学效率和教学 质量的提高。但是, 过度使用和依赖计算机辅助教学, 反 而会削弱教学效果。因此, 我们只能给它定位在“辅助”二 字上, 把它融合到传统的课堂教学中, 采取多媒体、网络 环境下学生自学, 教师导学和课堂面授三者相结合的形式, 既充分调动学生的主体性, 又充分发挥教师的主导作用, 只有这样才能取得最好的教学效果。计算机辅助教学的发
展前景广阔, 也对教师提出了前所未有的挑战, 教师必须 不断更新教学理念和教学方法、更新教育技术知识, 进行 终身学习。

\section{6. 致谢}

本项目受“中央高校基本科研业务费专项资金”资助。 (资助号：2014 MS180)

\section{参考文献(References)}

[1] Mingyuan Chen, Information Procession of the Language. Shanghai: Knowledge Press, 1982. (In Chinese).

[2] Stephen Krashen, Principles and practice in second language acquisition. Oxford: Pergamon, 1982.

[3] Merrill Swain and Sharon Lapkin, "Problems in Output and the Cognitive Processes They Generate: A Step Towards Second Language Learning”, Applied Linguistics, vol. 16, pp. 371-391, 1995.

[4] Haisen Zhang and Yan Li, "Study of blended learning and experimental teaching of English Listening in multimedia environment", Media in Foreign Language Instruction, pp.25-31, 2006(03). (In Chinese).

[5] Felicity O'Dell, "Helping teachers to use a self-access center to its full potential”, ELT, vol. 46, pp.153-159, 1992.

[6] Fansheng Meng, "CALL---the Role and Strategies of Computer Aided Language Learning", Media in Foreign Language Instruction, pp.32-35, 2005 (02). (In Chinese). 